\title{
Virtual Trial Room
}

\author{
Akshay Shirsat ${ }^{1}$, Samruddhi Sonimindia ${ }^{2}$, Sushmita Patil ${ }^{3}$, Nikita Kotecha ${ }^{4}$, Prof Shweta Koparde ${ }^{5}$ \\ Departmen Of Compute Engineering ${ }^{1,2,3,4,5}$, Savitribai Phule Pune University ${ }^{1,2,3,4,5}$ \\ Email: akshayshirsat43@gmail.com ${ }^{1}$,kotechanikita846@gmail.com ${ }^{2}$
}

\begin{abstract}
Augmented reality is the technology that expands our physical world, adding layers of digital information onto it. Augmented reality adds digital element to live view by using cameras or sensors. The main aim of this work is to develop virtual trial room using augmented reality which allows user to try on virtual clothes. Kinect sensor is used to calculate the effective distance between the sensor and the user who is standing in front of it. Based on this distance we can analyze how the clothes will look on user. This application improves present augmented based posing and gives full view of 3D image rather than 2D image.
\end{abstract}

Keywords- Kinect v2, Skeleton tracking, processing, Augmented reality

\section{INTRODUCTION}

A lot of shoppers have encountered a problem that trying clothes in clothing stores is usually a time consuming activity especially during peak hours such as weekends, it might not even be possible to try-on clothes in such cases as online shopping. Also Due to security reasons there is limitation on number of garments that can be taken for trial at a time (3). Also some times the shoppers are unable to show the customers the new stock that is supposed to arrive in coming few days. To overcome these problems we aim to develop a virtual trial room using augmented reality.

In this proposed application a sensor named Kinect is used for the overall analysis of the user's body measurements with the help of sensor information and his positioning coordinates. (5)

Following components will be required to design the system.

\subsection{Kinect sensor}

Kinect is a line of motion sensing input devices that was produced by Microsoft. Kinect has resolution of up to $1280 \times 1024$, monochrome depth sensing video stream is in VGA resolution with 11-bit depth. This sensor will give video frames as input to system for finding the available dresses in database [6].

\subsection{Processing}

Processing is an incredibly powerful and versatile programming environment and language. All Processing code is translated to java code first. This means you can write java code and import java libraries in your Processing code, within (or outside) the Processing IDE.

\subsection{LCD Screen}

LCD screen will be used as interface between user and system for displaying the output.

\section{LITERATURE SURVEY}

2.1. Md. Farhan hamid, md. Ashraful alam, "virtual wardrobe for physically impaired using microsoft kinect sensor"

This paper throws light particularly on physically impaired people who are partially or completely challenged. For completely impaired people, sensor senses the head movements for iterating through the clothes and the eyes movement for selection of apparel[5]But the paper tends to incline more towards the challenged people rather than normal people leading to noticeable change in functions adding to which the population too reduces as compared to normal people.

\subsection{Ari Kusumaningsih; Arik Kurniawati; Cucun Very Angkoso, Eko Mulyanto Yuniarno; Mochammad Hariadi, "User experience measurement on virtual dressing room of Madura Batik clothes"}

The system proposed in paper is used for online trial of Madura Batik clothes which is the regional wear of Indonesia. It provides virtual reality trial room for foreign customers to change consumer shopping experience and increase buying desire [8]. The major drawback here could be that the apparels are limited only to their regional wear and nothing apart from that.

\subsection{Srinivasan K.,Vivek S., "Implementation of virtual fitting room using image processing"}




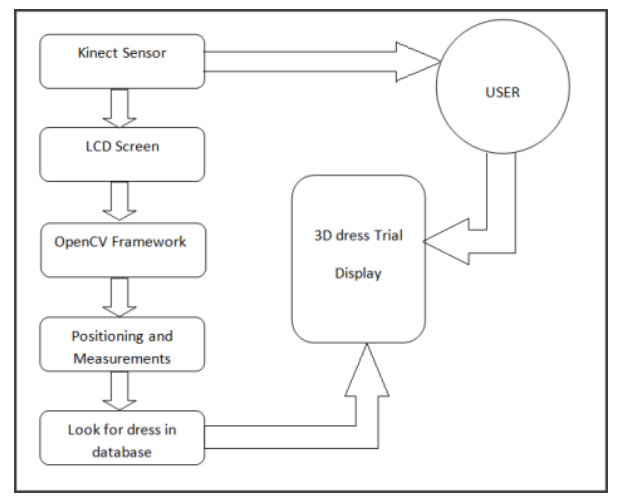

Fig. 1. System Architecture.

This system uses a fixed webcam for capturing the images of the user. This image is processed by MATLAB to compute every pixel of the image and check which apparel suits the best as per user's choice [9]. The drawback for this is that the system was only used for online shopping platform and not for any other offline use. Also as there is absence of kinect sensor due to which the actual measurements with respect to the depth is missing

\section{PROPOSED SYSTEM}

The implementation of the project starts by initializing the kinect sensor, which acts as the main hardware of the project. Also for running the application, the IDE(Integrated Development Environment) is Processing 3.5.3. The programming language used is Java. As soon as the application starts, the GUI that flashes a debut is the choice given the user to choose between male and female. Once the first step is done, the next step consists of Apparel selection which consists of different categories like shirt, pant, dress, t-shirt. Once that is selected then the selected apparel gets mapped onto user body with the help of body coordinates. This could virtual representation of look of the apparel.

To augment the cloth onto the virtual body in the virtual environment of user interface, we need certain calculated parameters as an input for modeling the cloth and augmenting the cloth onto the virtual body. To achieve this, Kinect V2 provides 25 joints tracked at 30 frames per second frequency and we can access the joints using the Kinect SDK. Out of the 25 joints, we use certain joints to calculate the measurements of shirt required to be augmented on the virtual body. The figure depicts the joints obtained by skeletal tracking with which we will be performing some calculations for modeling the shirt later in this paper. Since, we are calculating the RGB pixel value at a position, say $\mathrm{A}$, on user body by color stream and calculating the position coordinates by depth stream, we need to map each other to find the RGB pixel at a position $\mathrm{A}(\mathrm{x}, \mathrm{y}, \mathrm{z})$. But since color stream and depth stream have different resolutions, we need the use of Coordinate Mapper which maps corresponding coordinates correctly. As a result, we have access to all coordinates on virtual user body with corresponding RGB values. The image has been taken from official Microsoft site to show the various joints detected while skeletal tracking. To augment the shirt onto the virtual human body, we need to fill the color stream of the tracked user by pixels of shirt stream at positions where the shirt is supposed to be augmented onto the virtual body. For instance, to calculate the length of shirt, we make use of the hip center joint coordinates and shoulder center coordinates. To calculate the width of the shirt around shoulders, we make use of the shoulder left joint coordinates and shoulder right joint coordinates and their difference gives the width of shirt around the shoulder. To calculate the waist size, we use the hip left joint coordinates and hip right joint coordinates and their difference gives the waist size. These sizes can also help in scaling and cropping the shirt to anticipated measurement. For rotation, we make use of slope of line formed between shoulder center joint coordinates after rotation and hip center joint coordinates after rotation with the slope of the line formed by shoulder center joint coordinates before rotation and hip center joint coordinates before rotation to calculate the rotation angle.

Our object model is the geometrical object that created by using Blender3D software. We created models for both male and female consumer. 3-D meshes of clothing are designed in Blender3D software and imported to $\mathrm{Obj}$ and mtl file. In this research, Blender3D created Obj-files for the $3 \mathrm{~d}$ vertex modeling object, mtl-files for describing the surface shades.

Homogeneous transformation matrix for 3D bodies and $3 \mathrm{D}$ has been made. We use a rigid transformation which consists of rotation (R), translation (T) and scaling (S)[8]. These three values transform the rigid object from a three dimensional coordinate to the reference coordinate. The transformation of each joint point from one of the three dimensional coordinates to the other is transformed by the value of certain rotational, translational and scaling values. The most commonly used definition in graphics describes a rotation by Euler angles $(\Phi, \theta, \Psi)$ as a product of three rotations. The matrix $\mathrm{M}$ for the rotation is, therefore, product of three others [9]. 


\section{Scaling:}

Position of left-shoulder is calculated. This real world position is converted to projective. The same is repeated for right-shoulder To calculate $\mathrm{x}$ coordinate, distance between left and right shoulder position is calculated.
- $\quad \mathrm{x}$-coordinate=RightShoulderPos. $\mathrm{x}$ LeftShoulderPos.x.

- Neck position of skeleton=skel-neck.

- $\quad$ mid.y=((LeftHip.y+RightHip.y)/2)

- $\quad y$-coordinate $=\operatorname{sqrt}($ sq(skel-neck-mid.y $))$

- $\mathrm{z}$-coordinate is manually entered.

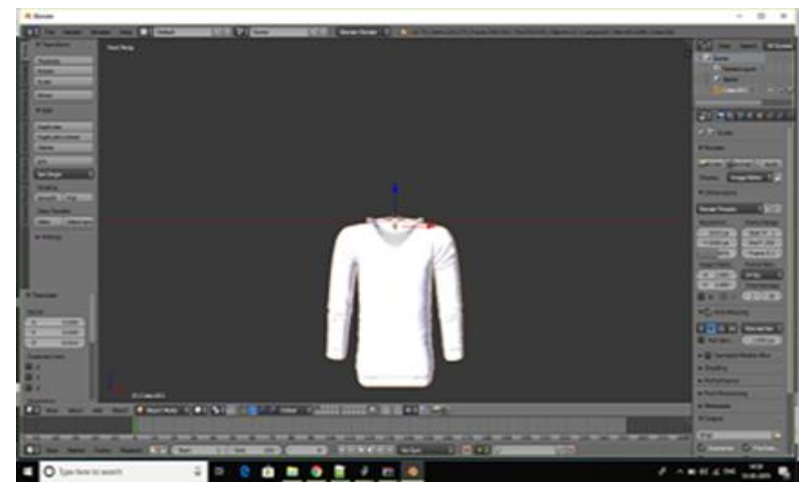

Fig. 2. Blender 3D.

\section{Translation:}

- The centre point of $3 \mathrm{~d}$ model= jointPos

- This real world position is converted to projective.

- $\quad$ translate(jointPos.x,jointPos.y,60)

- The model gets translated

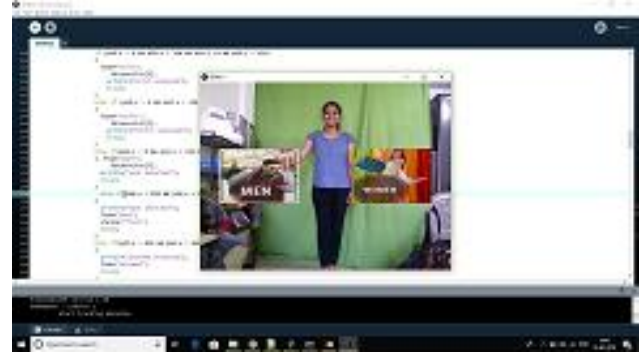

Fig 3. Output

\section{Rotation:}

To calculate rotation around $\mathrm{x}$ axis

- $\quad$ phi=atan(orientation.m12/orientation.m22) To calculate rotation around y axis

- theta=-asin(orientation.m02)

To calculate rotation around $\mathrm{z}$ axis

- $\quad \mathrm{psi}=\mathrm{atan}$ (orientation.m01/orientation.m00)

\section{CONCLUSION}

As the final output, system will display the available dresses from database according to positioning and measurements of user. The user can access the GUI by hand gesture and can select the apparel or also select various category. Thus it will be very convenient and hassle free for the customer to choose the perfect apparel through virtual means without much of inconvenience. Also an additional feature that gets displayed on the apparel would be 'S','M', 'L','XL'. Along with this the price of the product description about the selected category can also be displayed.

\section{REFERENCES}

[1] Yashwant Kanduri, Sanugula Durga Prasad, "A modular Approach for Cloth Modelling in Virtual Fitting Room", $201611^{\text {th }}$ International Conference on Industrial and Information systems(ICIIS)

[2] Anagaha Ramesh, Ankit Raj Kushal, Brinda D, Vaishnavi S, Purohit Shrinivasacharya, "3D Virtual Trial Room", Volume 6/Issue 13, International Journal of Engineering Research \& Technology(IJERT)

[3] Vinaya Kulkarni, Sailee Morde, Bhairavi Pawar, Supriya Mahadik, Rutuja Dahore, "2D Virtual Trial Room Using Augmented Reality", Volume 4/ Issue 1, International Journal on Future Revolution in Computer Science and Communication Engineering.

[4] Shreya Kamani, Neel vasa, Kriti Shrivastava, 
International Journal of Research in Advent Technology, Vol.7, No.5, May 2019

E-ISSN: 2321-9637

Available online at www.ijrat.org

"Virtual Trial Room Using Augmented Reality", Volume 3, Number 6, International Journal of Advanced Computer Technology (IJACT)

[5] Md. Farhan Hamid, Md. Ashraful Alam, "Virtual wardrobe for physically impaired using Microsoft Kinect Sensor",2017 IEEE $2^{\text {nd }}$ International Conference on signal and image processing

[6] [online] https://en.wikipedia.org/wiki/Kinect

[7] [online] https://en.wikipedia.org/wiki/OpenCV

[8] Ari Kusumaningsih; Arik Kurniawati; Cucun Very Angkoso, Eko Mulyanto Yuniarno; Mochammad
Hariadi, "User experience measurement on virtual dressing room of Madura Batik clothes", 2017 IEEE(978-1-5386-2182-0/17), 2017 International Conference on Sustainable Information Engineering and Technology

[9] Srinivasan K.,Vivek S., "Implementation of virtual fitting room using image processing" , 2017 IEEE International Conference on Computer, Communication and Signal Processing(ICCSP2017) 